\title{
On the development and early observations from a towing tank-based transverse wing-gust encounter test rig
}

\author{
S. J. Corkery ${ }^{1}$ (I) H. Babinsky ${ }^{1} \cdot$ J. K. Harvey ${ }^{1}$
}

Received: 20 March 2018 / Revised: 30 June 2018 / Accepted: 17 July 2018 / Published online: 4 August 2018

(c) The Author(s) 2018

\begin{abstract}
Small unmanned aerial vehicles and biological fliers can experience wind gusts of similar magnitude to the flight speed, which is detrimental to flight stability. For one encounter type, the low Reynolds number transverse gust, little is known about the fundamental fluid mechanics due in part to the difficulties in replicating the scenario experimentally or computationally. The aim of this work is thus to present the development and characterisation of an apparatus capable of generating and measuring the transient response of large amplitude transverse wing-gust encounters. The system is designed to produce a sharp-edged gust profile for direct comparison with the linear Küssner model. Particle image velocimetry (PIV) measurements show that the system successfully generated a steady top-hat shaped gust. A technique using inertial sensors has been used to minimise the effects of model vibration in measuring the unsteady forces. A wing-gust interaction with cross flow velocity equal to the flight speed is also presented. For this interaction, a strong leading edge vortex forms and vorticity of opposite sense is shed at the trailing edge. The trailing edge vorticity remains relatively planar, which is similar to the planar wake assumption of the Küssner model. Large deformation of the gust shear layers is visible upon wing entry, which is a deviation from the 'rigid' shear layers assumed by linear theory. Despite differences in flow topology between theory and experiment, the lift force coefficients match surprisingly well during entry into the gust, but deviate upon exit.
\end{abstract}

\section{Graphical abstract}

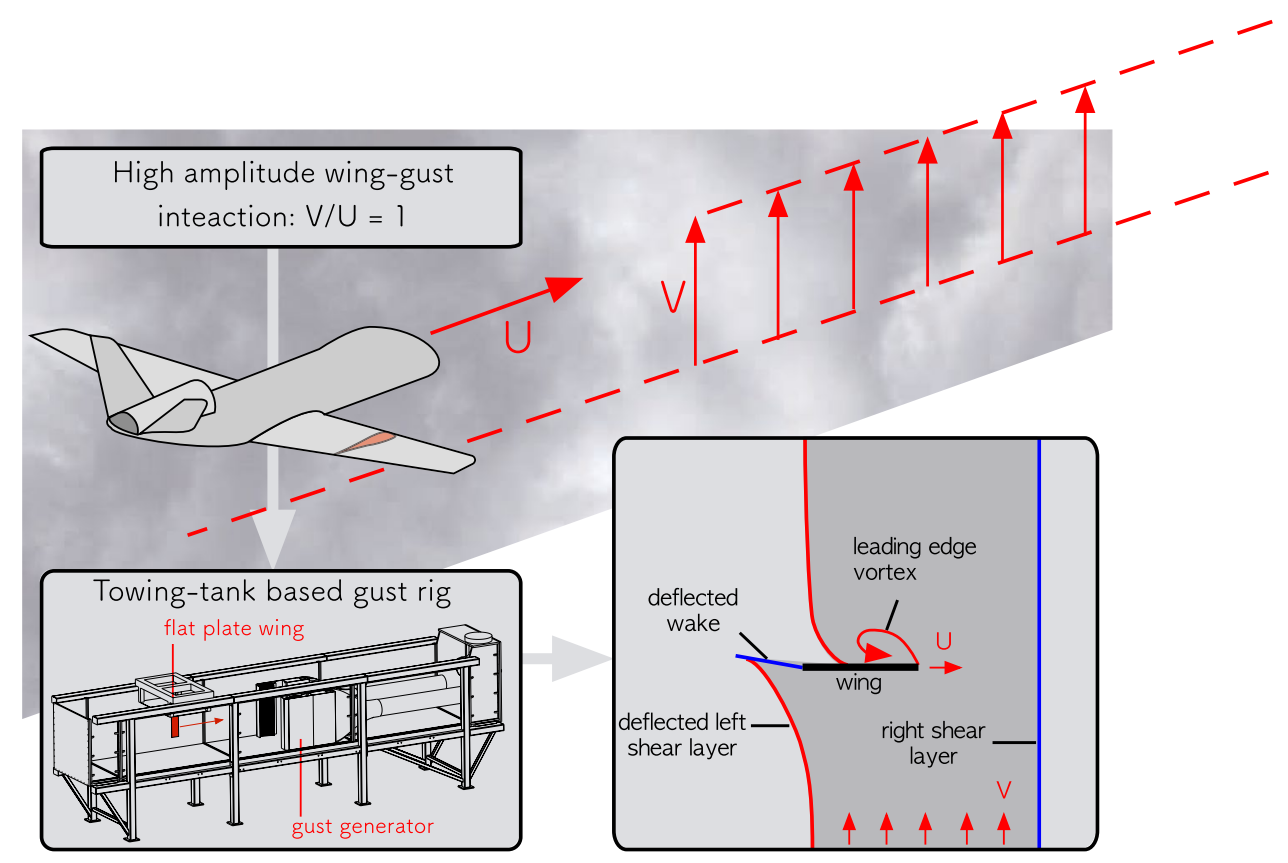

Extended author information available on the last page of the article 


\section{Introduction}

The development of small low-cost sensors and flight platforms has facilitated extensive growth in the use of unmanned aerial vehicles (UAVs) for military, commercial and consumer purpose. Applications include professional cinematography, surveillance and rapid transport of goods between geographical locations. While the small size of UAVs is beneficial from a cost and accessibility standpoint, herein lies a limitation from a flight stability and safety perspective. Small UAVs operate at altitudes well within the atmospheric boundary layer and fly across high shear and wake regions behind local obstructions (Watkins et al. 2006). In this domain, the wind turbulence intensity can exceed 50\% (Mohamed et al. 2015; Walshe 1972). Watkins et al. (2006) showed that for a typical radio aircraft sized fixed wing UAV, there can be rapid changes in angle of attack in excess of $25^{\circ}$, as well as span-wise variations in incidence of up to $15^{\circ}$ which result in roll instability. These problems are compounded with reducing vehicle size, as the optimal flight speed $(U)$ reduces with decreasing mass (Spedding and Lissaman 1998), thus the gust ratio $(V / U)$ increases for a given wind speed $(V)$. Consequently, the safe operating speed is reduced (Mohamed et al. 2015; Watkins et al. 2006, 2010; Spedding and Lissaman 1998; White et al. 2012) as well as arguably the reliability and usefulness.

One potential means to improve gust performance of UAVs is through onboard wind sensors and 'counter control' actuation using the lifting surfaces. A model of the response of the UAV to the disturbance is required to determine the correct control authority. There are, however, only limited direct measurements in the literature that characterise the response of an aerofoil to a large amplitude, low Reynolds number, and transverse wind gust. This is due perhaps to the experimental challenges faced with replicating a transverse gust encounter and historically work in this field has been directed toward the application of manned rotor or fixed wing aircraft. During forward flight of helicopter or tilt-rotor aircraft, the tip vortices from the upstream blades are advected downstream and can interact with the following blades. This is one example of a transverse wing-gust encounter as each blade passes through a region of free vorticity which induces up- or downwash velocity perturbations onto the wing. However, studies of the blade vortex interaction are typically limited to small velocity perturbations and Reynolds numbers in excess of 200,000 (Zanotti et al. 2014; Peng and Gregory 2015; Rival et al. 2010; Homer et al. 1993; Mai et al. 2011). For small angles and attached flow conditions, the phenomenological-based Küssner, Wagner, or Theodorsen models have been shown to fit

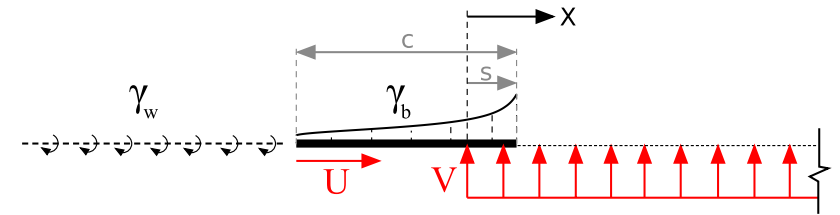

Fig. 1 Schematic of Küssner's problem Adapted from Leishman (2000)

measurements reasonably well (Patel 1978, 1982; Zaide and Raveh 2006; Leishman 1996, 1997). These models are linear and describe the effect of a kinematic or flow field disturbance on bound circulation. In one study by Perrotta and Jones (2017), large amplitude wing-gust encounters were tested using a free jet-like sine-squared velocity profile. The measurements were not, however, compared with the Küssner response for this gust profile. The accuracy of the Küssner model for a high amplitude gust encounter is thus unknown, nor is it known whether the mechanisms of lift production are equivalent.

An understanding into the mechanisms of high gust amplitude lift production may be attained from research on rapidly accelerating 'surging', 'pitching' or 'plunging' aerofoils at low Reynolds numbers. In contrast to the linear models, the bound circulation is small (Pitt Ford and Babinsky 2013) and the flow field may be dominated by vortices shed from the leading and trailing edges (LEV and TEV, respectively). Lift is generated though a combination of the change in impulse of the flow field due to growth and advection of the LEV and TEV, as well as due to classical added mass (Stevens and Babinsky 2017). It is hypothesised that similar lift production mechanisms apply to the large amplitude transverse gust case; but to address this first one requires a means to measure such encounter. The aim of this paper is to present and characterise a test apparatus capable of generating a sharp-edged gust. This profile is desired as it enables the assumptions of the Küssner model to be tested directly. The transient response of a single wing-gust encounter is additionally presented. The results are then compared with the most suitable simple theoretical description of such a flow, Küssner's gust model.

\subsection{Küssner's sharp edge gust model}

The Küssner model describes the growth in lift coefficient for a flat plate wing gradually entering a sharp-edged transverse gust as shown schematically in Fig. 1. The model assumes the gust to have a non-deformable shear layer and the wing is modelled as a broken line aerofoil (Von Karman and Sears 1938). The camber 'pivot point' and angle of incidence progressively changes upon gust entry, which leads to a change in bound circulation $\left(\gamma_{\mathrm{b}}\right)$. 


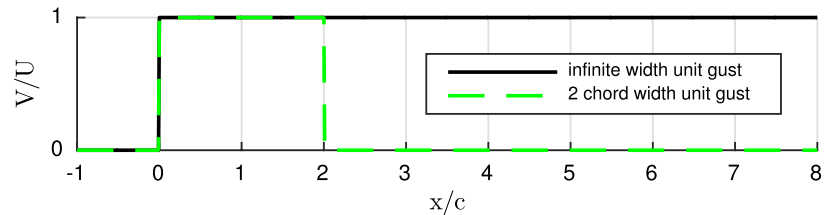

(a) Gust velocity with position

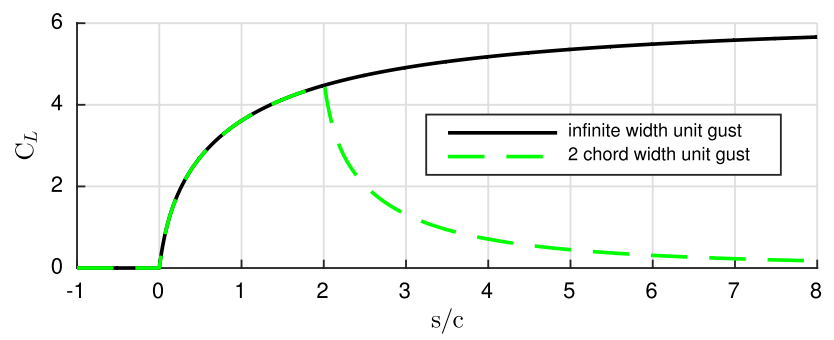

(b) Lift coefficient with position

Fig. 2 Response to a unit gust of infinite extent and finite width of two chord lengths

As circulation must be conserved, vorticity is shed into the wake at the trailing edge $\left(\gamma_{\mathrm{w}}\right)$ which delays growth in lift due to downwash induced back onto the aerofoil. The general lift coefficient is given by Eq. (1),

$C_{\mathrm{L}}=2 \pi \frac{V}{U} \psi(s / c)$

where $\psi(s / c)$ is the Küssner response function, $s$ is the distance from the wing leading edge to the rising gust edge and $c$ is the wing chord. While the true Küssner solution has to be calculated iteratively, Eq. 2 gives an approximation for $\psi(s / c)$ for an infinite width gust by Bisplinghoff et al. (1955). The corresponding change in lift coefficient for a unit gust $(V / U=1)$ is shown in Fig. 2.

$\psi(s / c)=\frac{4(s / c)^{2}+2(s / c)}{4(s / c)^{2}+5.64(s / c)+0.80}$.

The response to any other arbitrary gust profile can be calculated by means of Duhamel's integral (Leishman 2000). The response is found by decomposing the input function $(V / U=f(x / c))$ into a series of impulses, for each of which a change in lift coefficient is calculated in accordance to Eq. 1. The total response is thus determined by linear superposition of the response of all past impulses. For this work, we will consider a finite gust width of two chord lengths, for which Küssner's model predicts a peak change in lift coefficient of approximately $70 \%$ of the infinite width gust case as shown in Fig. 2.

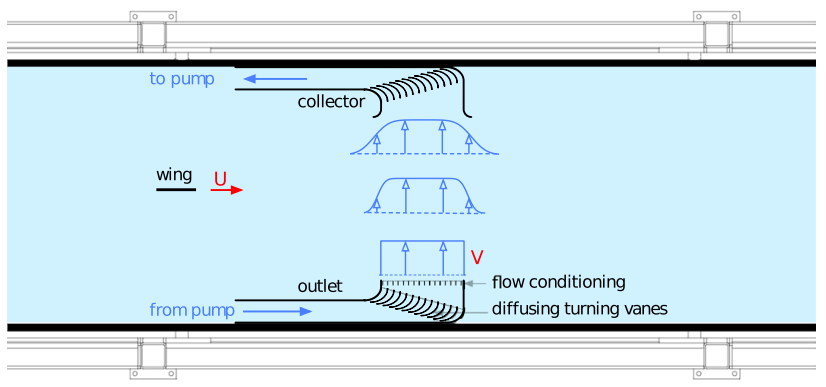

Fig. 3 Schematic of the gust setup inside the tank. View from above the test section

\section{Experimental setup}

\subsection{Gust rig}

Conventional wind tunnel-based gust rigs introduce a transverse velocity perturbation by typically one of two means. One method uses a pair or cascade of actuated vanes upstream of the model to 'turn' the incoming flow (Tang and Dowell 2010; Saddington et al. 2014; Brion et al. 2015; Patel and Hancock 1977; Tang et al. 1996; Ham et al. 1974; Patel 1982). However, this can be limited to gust ratios of approximately 0.3 due to flow separation on the vanes, coupling of the free stream velocity with vane deflection angle and increased turbulence levels. The other method first proposed by Ryan and Dominy (2000) and adapted by Volpe et al. (2013) for the study of road vehicles in cross wind, can be described as a double wind tunnel where a transient jet of air is introduced from the side of a primary wind tunnel at an angle to the free stream. Flow from the secondary tunnel is introduced using a cascade of shutter vanes that are sequentially opened at a rate equal to the free stream. In the experiments conducted by Ryan and Dominy (2000) sharpedged gusts were achieved, however, the cross flow uniformity and flow quality is questionable with significant velocity under- and overshoots from the intended square wave profile.

To overcome the challenges of wind tunnel-based cross flow apparatus, a system was specially designed for the test section of the Cambridge University Engineering Department (CUED), a $9 \times 1 \times 1 \mathrm{~m}$ towing tank. As schematically shown in Figs. 3 and 4, a pump and ducting system is used to create a region of conditioned cross flow in the tank through which the wing model is towed.

Conducting the experiment in a towing tank has two primary advantages. First, water is used as the working fluid to take advantage of Reynolds number scaling effects, whereby flow speeds are lower and forces are approximately four times greater compared to the equivalent flow conditions in air. Particles can more readily be suspended in the fluid for particle image velocimetry and dye may be injected for flow visualisation. Second, unlike in a wind tunnel, the flow 


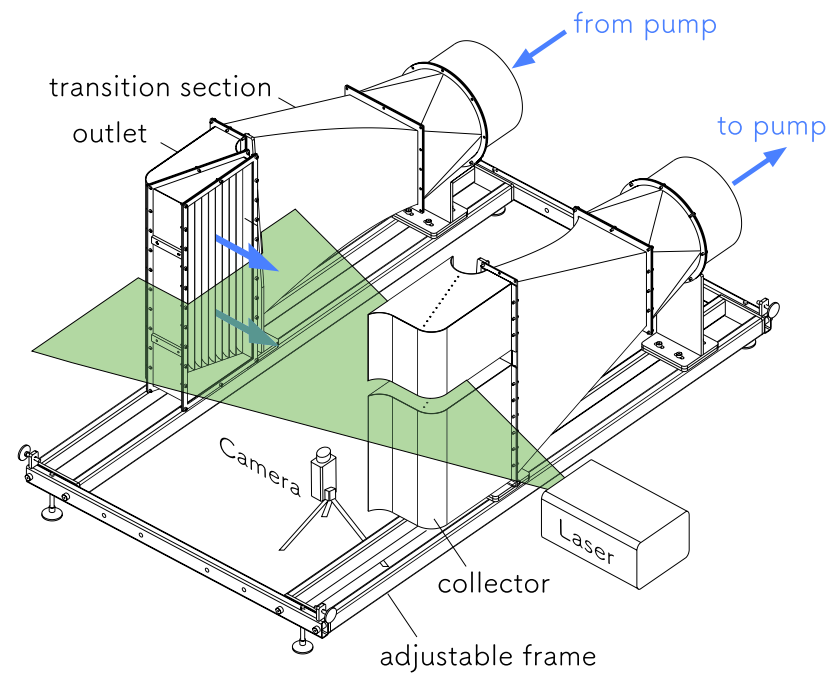

Fig. 4 Gust ducting and PIV configuration

field is directly representative of an aircraft or UAV in flight, i.e., the fluid is at rest while the model follows a prescribed kinematic profile. A transverse gust can thus be replicated by simply creating a velocity perturbation at a 'fixed' location in space using a pump as actuation. Humphreys (1995) developed a similar technique for cross wind studies on lorry and railway vehicles in which a towed model is propelled to pass through the flow of an open jet wind tunnel. The technique has additionally been used by Perrotta and Jones (2017) in a water towing tank. The authors, however, created a jet-like sine-squared profile and not the sharp-edged top-hat profile this work is looking to create.

The outlet velocity profile from the CUED gust rig has a number of controls. The flow velocity is set using a 2.2 $\mathrm{kW}$ continuously variable speed pump to enable independent control of the cross flow velocity relative to the wing. This allows gust ratios from 0 to over 1 to be generated. From the pump, the flow is passed through a constant area transition section to the outlet section. In the outlet section, flow is redirected by $90^{\circ}$ such that it can exit the outlet ducting in the direction perpendicular to the carriage path. This requires the flow to be diffused with an expansion ratio of approximately 3 from the pump, which has a $250 \mathrm{~mm}$ diameter circular duct, to the outlet geometry of $240 \mathrm{~mm}$ width and $600 \mathrm{~mm}$ height. Within the confines of the tank, there is insufficient space to diffuse the flow prior or after the turning vanes. To overcome this constraint, diffusing turning vanes are utilised. Without boundary layer control diffusing turning vanes have been shown to have total pressure losses similar to that of standard turning vanes without diffusion (Friedman and Westphal 1952), although the maximum expansion ratio tested in literature is 1.45 . The behaviour of a cascade of diffusing turning vanes with area ratio of 3 is thus unknown, but as shown in Fig. 5a, flow separation

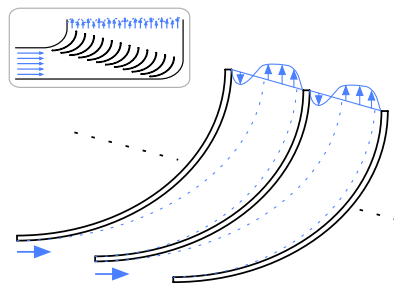

(a) without screen

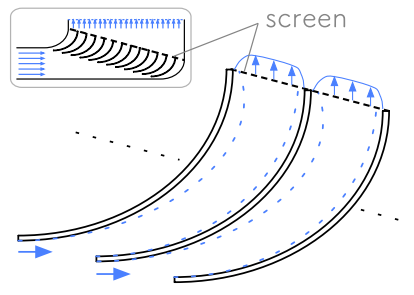

(b) with screen
Fig. 5 Flow from diffusing turning vanes with and without outlet screen

leading to non-uniformities and unsteadiness is likely due to the severely adverse pressure gradients imposed on boundary layer over the vanes.

Flow control similar to that discussed by Mehta and Bradshaw (1979) for wide angle diffusers is thus utilised, where wire mesh screens are stationed in the diffuser to encourage flow reattachment and uniformity. According to the guidelines by Mehta and Bradshaw (1979), a pressure drop coefficient of $K=\Delta P / \frac{1}{2} \rho U^{2}=2$ will suffice for an expansion ratio of 3. It is desirable to use multiple lower $K$ screens distributed through the diffuser, however, this is unpractical given the geometric constraints and thus a single high $\mathrm{K}$ screen was placed at the vane outlet plane (Fig. 5b). Following the vanes, the velocity profile is 'conditioned' using an aluminium honeycomb ( $3.2 \mathrm{~mm}$ cell width) and a mesh screen $(K=8.55$ at $0.5 \mathrm{~m} / \mathrm{s})$ to reduce transverse and axial velocity perturbations prior to entry into the tank. Spatially variable resistance screens could be installed to generate non-square wave outlet profiles, but these are not used here. Downstream of the outlet, the return circuit of the pump is used to 'collect' the gust using a similar set of turning vanes as used within the outlet, however, no screens are required as the flow is entering a gradual contraction. The jet was 'collected' to minimise both the turbulence in the 'still' regions of the tank by preventing the majority of the jet from mixing and the transport of fluid toward the pump inlet which would be located elsewhere in the tank. It is shown later in Sect. 4 that unsteady shear layers are developed on the edges of the jet, but there is comparatively little disturbance to the ideally quiescent flow regions either side of the gust as measured over time periods typical to that of test run (approximately $30 \mathrm{~s}$ from startup). Finally, the gust outlet and collector are mounted to an adjustable frame that allows the spacing of the outlet and collector to be varied between 240 and 600 $\mathrm{mm}$ in $120 \mathrm{~mm}$ increments. This is to enable the wing to be moved closer to the outlet to adjust the 'sharpness' of the gust shear layers at the wing entry point, as well as to enable the study of blockage effects on the response of the wing.

The wing model used here is a carbon fibre flat plate with $120 \mathrm{~mm}$ chord, $480 \mathrm{~mm}$ length and $4 \mathrm{~mm}$ thickness. Based 
Table 1 Contributions to PIV measurement error (pixels)

\begin{tabular}{llllll}
\hline$\sum \varepsilon_{\mathrm{PIV}}$ & $\varepsilon_{\text {bias }}$ & $\varepsilon_{\mathrm{rms}_{0}}$ & $\varepsilon_{\mathrm{rms}_{\delta}}$ & $\varepsilon_{\mathrm{rms}_{\rho}}$ & $\varepsilon_{\mathrm{rms}_{i}}$ \\
\hline 0.135 & -0.01 & 0.01 & 0.01 & 0.025 & 0.10 \\
\hline
\end{tabular}

on wing chord $(c)$ and cross flow velocity $(V)$, the setup gives a working Reynolds number range up to a maximum of 50,000 with the gust rig operating at full capacity. The gust has a relative width of 2 chords, which as discussed in Sect. 1.1 according to Küssner will capture $70 \%$ of the transient response before the leading edge of the aerofoil reaches the 'back' gust shear layer.

\subsection{PIV}

The setup of the PIV laser and camera system relative to the gust outlet is shown in Fig. 4. A dual cavity Nd:YLF laser $(527 \mathrm{~nm})$ casts a light sheet horizontally through a gap in the collector module. This is set up to intersect the wing at the mid span location. A single Phantom Miro 310 camera is positioned underneath the tank and $\mathrm{TiO}_{2}$ particles were photographed at a rate of $200 \mathrm{~Hz}$. Every second frame was processed (frames 1-3, 3-5, etc.) with deformable interrogation windows of nominal size $12 \times 12$ pixels with $50 \%$ overlap. The total cross correlation error (in pixels) for each interrogation window can be represented by,

$\sum \varepsilon_{\mathrm{PIV}}=\varepsilon_{\mathrm{bias}}+\varepsilon_{\mathrm{rms}_{0}}+\varepsilon_{\mathrm{rms}_{\delta}}+\varepsilon_{\mathrm{rms}_{\rho}}+\varepsilon_{\mathrm{rms}_{i}}$,

where $\varepsilon_{\text {bias }}$ is consistent bias error due to loss of particle image pairs from out of plane motion (Raffel et al. 2007; Nobach and Bodenschatz 2009). The other error sources operate in a random manner and are attributed to particle image diameter $\varepsilon_{\mathrm{rms}_{0}}$, particle image displacement $\varepsilon_{\mathrm{rms}_{\delta}}$, interrogation window particle density $\varepsilon_{\text {rms }_{\rho}}$ and variation in particle image intensities $\varepsilon_{\text {rms }_{i}}$. Estimations for each parameter are given in Table 1 . The values, with exception of the particle image intensity term $\left(\varepsilon_{\mathrm{rms}_{i}}\right)$ are based on the work of Raffel et al. (2007) who quantified error sources through simulations with synthetically generated particles. The particle image intensity term is based on the work of Nobach and Bodenschatz (2009) who showed that an error on order of 0.1 pixels occurs due to variation in light intensity of particles displaced in the direction perpendicular to the laser plane. The mean particle displacement based on the gust velocity is 4.28 pixels, thus with $\varepsilon_{\mathrm{PIV}}=0.135$, the error is $3.15 \%$. Force and PIV measurements of wing-gust encounters are averaged over 5 runs. Since random error reduces with $1 / \sqrt{N}$ (Adrian and Westerweel 2011), where $N$ is the number of samples, the random error after batch averaging is approximately $1.5 \%$. The tank is left to settle for $15 \mathrm{~min}$

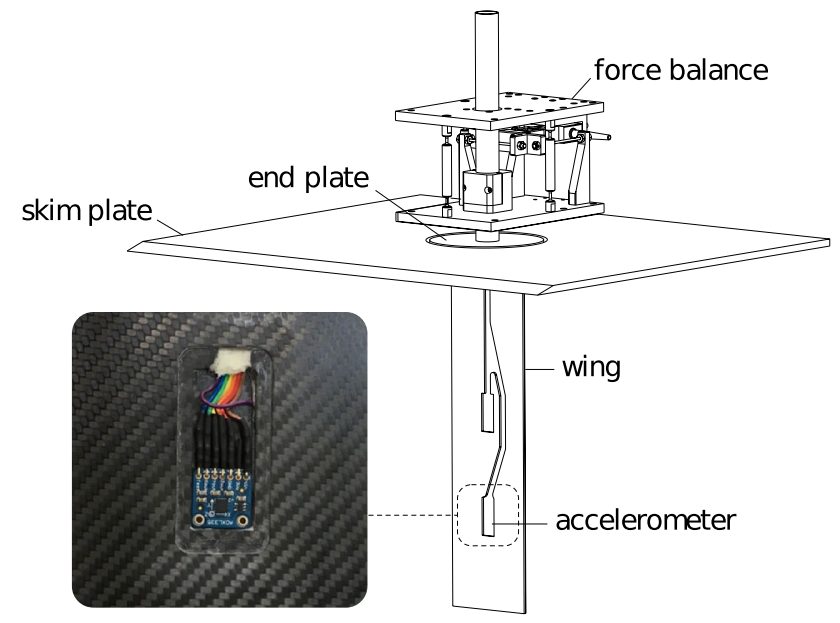

Fig. 6 Wing and balance configuration

between each test run, after which the turbulence level has decayed below the minimum quantifiable threshold of the PIV system (Jones and Babinsky 2011).

\subsection{Force and inertial measurement systems}

Lift and drag forces on the wing were measured using the two-component $50 \mathrm{~N}$ strain-gauge force balance shown in Fig. 6. Each channel has a resolution of $0.01 \mathrm{~N}$. The maximum cross-talk between each balance channel is less than $0.5 \%$ of the full load range. This was quantified by applying known loads in the direction of each sensor (independently) and recording the cross-talk error on the unloaded channel. The 'moment' error due to offset loading of the wing relative to the balance sensors is less than $2 \%$ at $0.5 \mathrm{~m}$. This was quantified by applying a fixed load at increasing offset distances from the balance. The stated value represents a maximum error as the load offset exceeds the wing length. The total error in the measured force therefore has a maximum of $2.5 \%$ of the full scale signal. The balance is mounted directly to the tank carriage such that the balance sensors are in fixed alignment with the lift and drag directions, while the angle of incidence of the wing is adjusted relative to the balance.

A $180 \mathrm{~mm}$ diameter end plate is located at the top wing tip, which is set flush into the skim plate spanning the width of the tank. The skim plate is attached rigidly to the carriage just below the water line and suppresses surface wave effects in the vicinity of the wing. There is a $5 \mathrm{~mm}$ radial gap between the end and skim plates to circumvent the transmission of forces from the skim plate to the force balance. The arrangement produces a mirror image of the flow and wing, thus giving the wing an effective aspect ratio of 8 . The design additionally avoids the requirement for a tip clearance gap between the wing and skim plate, which has been suspected of introducing three dimensional effects that are 
detrimental to span-wise coherence of a leading edge vortex (Ol and Babinsky 2016; Son et al. 2016).

Embedded within the carbon wing is an ADXL335 3-component micro-electromechanical accelerometer. The wiring to the accelerometer runs internally through the wing for minimal flow disturbance. The sensor can measure accelerations up to $\pm 3 \mathrm{~g}$, has a nonlinearity of less than $\pm 0.3 \%$ and cross-sensitivity of $\pm 1 \%$. A second accelerometer is located on the towing tank carriage. The velocity and position of the carriage is calculated by numerically integrating the acceleration measured using the carriage mounted accelerometer. Gradual 'drift' in velocity and position due to cumulative sensor error is corrected by measuring the absolute position given by a separate quadrature linear encoder with $1 \mathrm{~mm}$ resolution. Combined position accuracy is estimated to be of order of $0.01 \mathrm{~mm}$. Data is recorded using LabView and a National Instruments 14 bit data acquisition card at a sampling frequency of $5 \mathrm{kHz}$.

\section{Inertial force decomposition}

Vibration due to flexibility of a load cell, sting or model can add significant noise to force measurements. This noise is particularly detrimental at low Reynolds numbers, whereby forces of interest can be just a fraction of a Newton. Filtering is often employed to smooth over vibrations (Granlund et al. 2011; Jones and Babinsky 2010, 2011; Pitt Ford 2013; Stevens 2013), however, this distorts transient forces by reducing their peak and increasing bandwidth (Pitt Ford 2013). The necessity for filtering can therefore limit the usable frequency range of force measurements to below the lowest mode of vibration of the system. This is problematic for the relatively sharp edge gust experiment conducted here as the expected frequency components of the transient fluctuations in force are above the natural frequency of the wing.

An additional source of measurement error results from the model and force balance self mass. During regions of carriage acceleration, an inertial force is applied though the load cell to accelerate the wing and balance mass. This force component is often quantified by taking the average of a series of 'tare' results from identical kinematic test runs conducted in air. The tare experiment takes advantage of the approximately 850 times difference in density between air and water, thus isolating the inertial component as fluid-dynamic forces are comparatively negligible. This method is not, however, without fault. Direct subtraction of the inertial force from each 'wet' run introduces additional noise due to a mismatch in resonant vibration frequencies between the dry and wet tests. The mismatch is a result of the increase in apparent mass of the model in water and subsequent reduction in natural frequency. For high Reynolds number testing of large models (such as those utilised for the study of vehicle aerodynamics), further

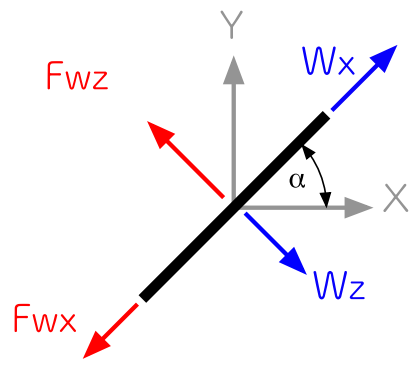

Fig. 7 Coordinate systems and balance applied force components

error in kinematics can be introduced as the carriage actuator responds differently due to increased model loading when in water. It is therefore desirable to improve the means by which both model vibration and the self mass force are rejected.

In the following sections, the equations of motion for the wing are derived to show that the use of accelerometer sensors enables inertial forces on the wing to be directly quantified. As model vibration and self mass are inertial forces, these can therefore be directly subtracted from the force measurements. This enables fluid dynamic-derived force contributions to be isolated with increased resolution and accuracy.

\subsection{General equations of motion}

First, we will consider the equations of motion for a flat plate wing undergoing arbitrary planar translation. As shown in Fig. 7, a wing coordinate frame $W_{x}-W_{z}$ is set at an arbitrary angle $\alpha$ to a global $X-Y$ coordinate. The fluid is assumed to be stationary at infinite distance from the $X-Y$ coordinate origin.

In a viscous flow, the forces acting on a body can be deconstructed into two components. The first is attributed to body motion in an irrotational fluid and the second is due to vorticity in the flow field about the body (Eldredge 2010; Graham et al. 2017). Acceleration of the wing in the direction perpendicular to its chord causes an added mass force, which is realised by a pressure difference across the plate surface. If the plate is assumed infinitely thin the added mass force must act perpendicular to the surface. For a flat plate wing with chord length $c$ and length $h$, the mass component is equivalent to the mass of a cylindrical volume of fluid with a diameter of one chord $\left(m_{v t}=\rho h \frac{\pi c^{2}}{4}\right)$. All (other) forces attributed to vorticity in the flow field are to be identified as one component $F_{\text {other }}$, which is typically the force of interest. The wing and balance system have mass $m_{w}$.

The forces applied by the balance to the wing in the $W x$ and $W z$ directions are,

$F_{w x}=m_{w} a_{w x}-F_{\text {other }, w x}$,

$F_{w z}=\left(m_{w}+m_{v t}\right) a_{w z}-F_{\text {other }, w z}$,

where the subscripts $w x$ and $w z$ defines values in the $W x$ and $W z$ directions, respectively. As shown in Fig. 7, the force 
measured by the load cell acts in an equal and opposite direction to that applied to the wing. Resolving the components of force on the balance in the $X-Y$ coordinate frame gives Eqs. (6) and (7). The terms $F_{\text {other }, X}$ and $F_{\text {other }, Y}$ are the transformed equivalent of $F_{\text {other,wx }}$ and $F_{\text {other,wz: }}$ :

$F_{b, X}=-\left(m_{w}+m_{v t}\right) a_{w z} \sin \alpha-m_{w} a_{w x} \cos \alpha+F_{\text {other }, X}$,

$F_{b, Y}=\left(m_{w}+m_{v t}\right) a_{w z} \cos \alpha-m_{w} a_{w x} \sin \alpha+F_{\text {other }, Y}$.

For the experiments at hand the wing is constrained in the $Y$ direction by the rails of the tank and therefore the wing can only experience accelerations in the $X$ direction. The $X$ direction acceleration, $a_{\text {car }}$, is directly measured with a carriage-mounted accelerometer. Provided the carriage is exceedingly stiff, the difference in measured acceleration and that physically experienced by the balance will be negligible regardless of the accelerometer position, as high-frequency vibrations relative to the two will be later filtered. It is best, however, to fix this accelerometer to the non-sensing side of the force balance. The latter was done for the present experiments. Resolving $a_{\mathrm{car}}$ into the wing coordinate system gives:

$a_{w x}=a_{\mathrm{car}} \cos \alpha$,

$a_{w z}=a_{\mathrm{car}} \sin \alpha$.

Substituting Eqs. (8) and (9) into (6) and (7) gives the forces applied to the force balance due to the prescribed body motion and flow field vorticity.

$F_{b, X}=-a_{\text {car }}\left(m_{v t} \sin ^{2} \alpha+m_{w}\right)+F_{\text {other }, X}$,

$F_{b, Y}=m_{v t} a_{\mathrm{car}} \sin \alpha \cos \alpha+F_{\mathrm{other}, Y}$.

\subsection{Modal vibration}

Here we derive an additional force component due to structural vibration, or rigid body motion of the test model relative to the prescribed motion of the force balance. It is desirable to make the model, carriage and balance system as stiff as possible to keep vibration forces to a minimum, so the aim of the proposed technique is not to be perfect in all scenarios, but simply to make good, or acceptable data better. In the instance of structural vibration, such as that of the relatively thin cantilever design flat plate wing utilised in this study, for any given forcing disturbance applied there is an infinite number of resonant frequencies and vibration mode shapes that may be excited. Typically low pass filtering is utilised to remove all vibration frequency content. Here we propose the use of the three axis accelerometer shown previously in Fig. 6 to quantify forces on the balance associated with the first mode vibration, such that subsequent low pass filtering can be applied at a higher frequency to preserve transient fluid-dynamic forces. For the wing model, submerged in water, the resonant frequencies of the first and second vibration modes are approximately 5 and $31 \mathrm{~Hz}$, respectively, thus the frequency response may be significantly improved if forces attributed to the first mode may be quantified. Assuming that the body has only one mode of vibration, or all frequency modes higher than the first are filtered, then the acceleration due to vibration $\left(a_{v}\right)$, is the difference between the carriage and wing accelerations.

$a_{v, w x}=a_{w x}-a_{\mathrm{car}} \cos \alpha$,

$a_{v, w z}=a_{w z}-a_{\mathrm{car}} \sin \alpha$.

The vibration accelerations $a_{v, w x}$ and $a_{v, w z}$ are dependent on the position of each accelerometer on the body. They must therefore be located such that they are away from the nodal points for the vibration mode to be compensated for. The accelerometer in the wing model shown in Fig. 6 is thus located toward the wing tip where it will have high acceleration associated with the first mode. Assuming the body is vibrating with simple harmonic motion, the force applied by the balance to the wing to accelerate the sensor in the $W x$ and $W z$ directions is:

$F_{v, w x}=m_{v x} a_{v, w x}$,

$F_{v, w z}=m_{v z} a_{v, w z}$.

The mass terms $\left(m_{v x}\right.$ and $\left.m_{v z}\right)$ are modal or effective masses as the entirety of the model will not experience the same accelerations as that measured with wing-embedded accelerometer. It is important to note that the modal masses are in essence a scaling factor that converts an acceleration measured at some point on the model, to a force applied to the model by the balance. It need not be equal to the sum of the physical and virtual masses of the body, but will scale proportionally with these terms. The modal masses can be found through an initial calibration to be described in Sect. 3.4. Resolving the vibration forces into the $X-Y$ directions gives:

$$
\begin{aligned}
F_{v, X}= & -m_{v z} \sin \alpha\left(a_{w z}-a_{\mathrm{car}} \sin \alpha\right) \\
& -m_{v x} \cos \alpha\left(a_{w x}-a_{\mathrm{car}} \cos \alpha\right), \\
F_{v, Y}= & m_{v z} \cos \alpha\left(a_{w z}-a_{\mathrm{car}} \sin \alpha\right) \\
& -m_{v x} \sin \alpha\left(a_{w x}-a_{\mathrm{car}} \cos \alpha\right) .
\end{aligned}
$$

\subsection{Combined force}

The total force applied to the load cell is the sum of the mean acceleration and vibration components. Thus,

$$
\begin{aligned}
F_{X}= & F_{b, X}+F_{v, X} \\
= & -\underbrace{a_{\text {car }} m_{v t} \sin ^{2} \alpha}_{\text {virtual mass }}-\underbrace{a_{\text {car }} m_{w}}_{\text {self mass }}+\underbrace{F_{\text {other }}, X}_{\text {remainder }} \\
& -\underbrace{m_{v z} \sin \alpha\left(a_{w z}-a_{\text {car }} \sin \alpha\right)-m_{v x} \cos \alpha\left(a_{w x}-a_{\text {car }} \cos \alpha\right)}_{\text {vibration }},
\end{aligned}
$$




$$
\begin{aligned}
F_{Y}= & F_{b, Y}+F_{v, Y} \\
= & \underbrace{m_{v t} a_{\mathrm{car}} \sin \alpha \cos \alpha}_{\text {virtual mass }}+\underbrace{F_{\text {other }}, Y}_{\text {remainder }} ; \\
& +\underbrace{m_{v z} \cos \alpha\left(a_{w z}-a_{\mathrm{car}} \sin \alpha\right)-m_{v x} \sin \alpha\left(a_{w x}-a_{\mathrm{car}} \cos \alpha\right)}_{\text {vibration }} .
\end{aligned}
$$

Equations (18) and (19) show that through application of potential theory and select placement of inertial sensors, the measured force can be deconstructed into virtual mass, self mass and vibration components. The remaining component encompasses forces arising from vorticity in the flow field. As we are uninterested in force contributions due to the model self mass and vibration, we can simply subtract these components to leave the virtual mass and viscous forces of interest.

\subsection{Calibration}

The mass components $m_{w}, m_{v t}, m_{v x}$ and $m_{v z}$ can be readily found. Calibration of $m_{w}$ was performed simply through turning the balance on the side such that the wing and balance self weight force due to gravity acts in the direction of the balance sensors $\left(m_{w}=\Delta F / \Delta a\right)$. The virtual mass $m_{v t}$ is calculated assuming the two dimensional potential flow solution, while the modal masses were found by setting the wing to an angle of incidence of $45^{\circ}$ and subjecting the towing tank carriage to a nominally 'impulsive' velocity profile while in water (change in displacement of $10 \mathrm{~mm}$ with high acceleration rates). An angle of $45^{\circ}$ is used to induce free vibration in both the $W x$ and $W z$ directions and is similar to the gust ratio of an experiment presented in Sect. 5 whereby a change in incidence of $45^{\circ}$ is experienced across the span of the wing. The velocity profile is shown in Fig. $8 \mathrm{a}$ and the deconstructed force response in the $X$, or negative drag direction is shown in Fig. 8d. After the impulse (from $20 \mathrm{~s}$ and onward) the tank carriage is at rest with negligible acceleration $\left(a_{\text {car }}=0\right)$ and the wing freely vibrates $\left(a_{v, w x}=a_{w x} \neq 0\right.$ and $\left.a_{v, w z}=a_{w z} \neq 0\right)$. Assuming non-bound vorticity in the flow field is negligible, the masses $m_{v z}$ and $m_{v x}$ are found by solving Eqs. (14) and (15). The acceleration terms $a_{v, w x}$ and $a_{v, w z}$ are measured directly by the wing-embedded accelerometer and are shown in Fig. 8b. The vibration forces in the wing coordinate frame are $F_{v, w x}=-\left(F_{X} \cos \alpha+F_{Y} \sin \alpha\right)$ and $F_{v, w z}=-\left(F_{X} \sin \alpha-F_{Y} \cos \alpha\right)$, thus can be determined from the forces $F_{X}$ and $F_{Y}$ measured by the balance. These are given by Fig. $8 \mathrm{c}$. Here the modal mass terms were averaged between the time of 20-21 s with a sample rate of 5 $\mathrm{kHz}$. Given the balance and accelerometer error is of order $2 \%$, post averaging the mass error is a negligible $0.03 \%$. Each mass parameter for the calibration is given in Table 2.

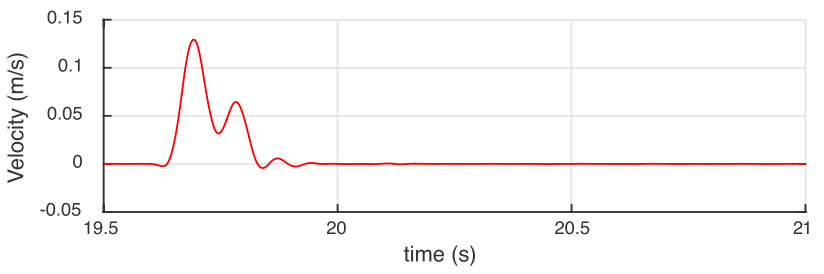

(a) Velocity profile

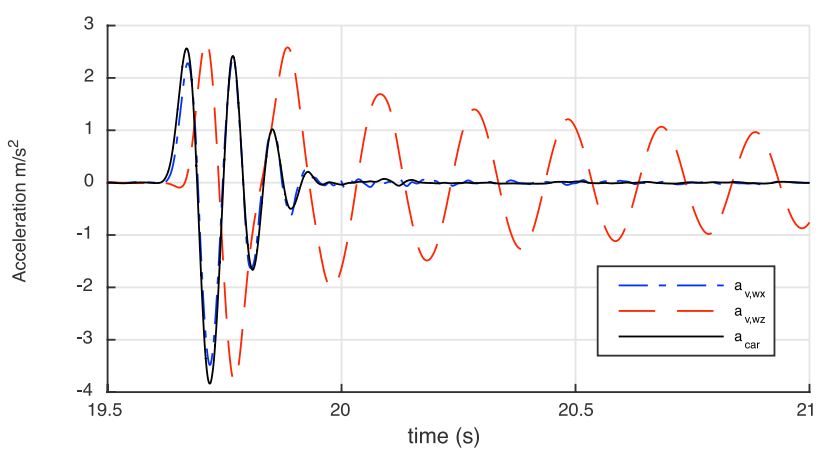

(b) Accelerometer measurements

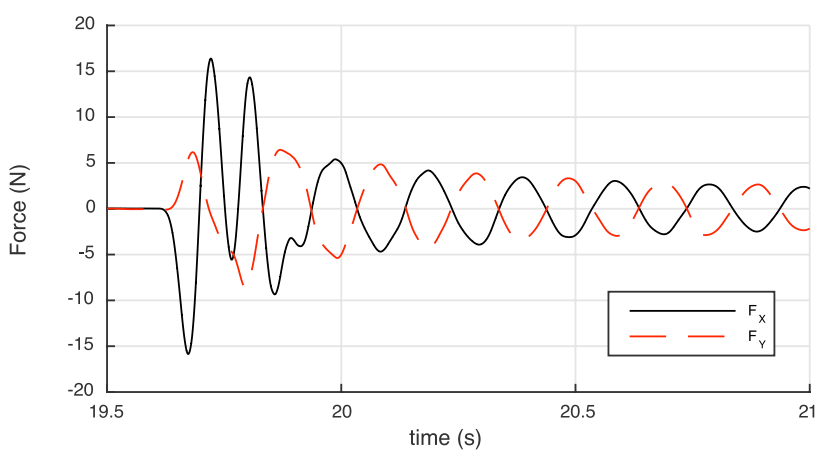

(c) Force balance measurements

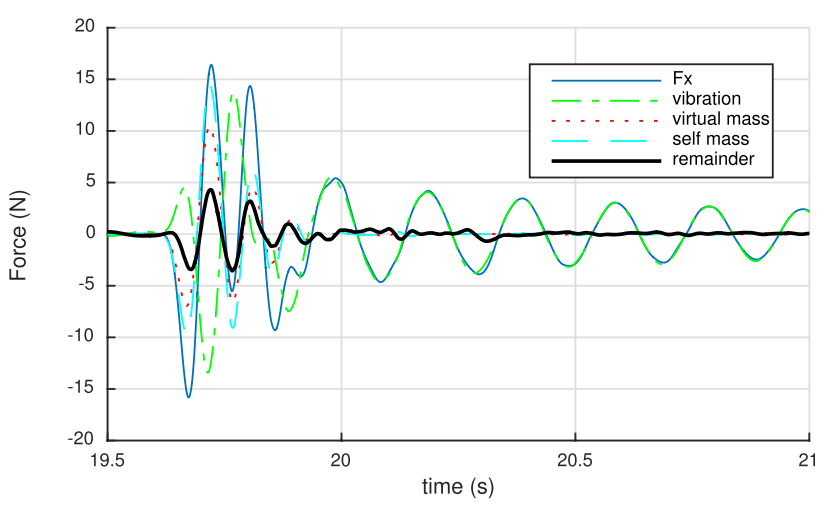

(d) X direction force decomposition (-drag)

Fig. 8 Calibration of inertial force system. A moving average with a period of $0.05 \mathrm{~s}$ is applied to smooth high frequency noise

Table 2 Mass parameters $(\mathrm{kg})$

\begin{tabular}{llll}
\hline$m_{w}$ & $m_{v x}$ & $m_{v z}$ & $m_{v t}$ \\
\hline 3.75 & 1.685 & 3.85 & 5.43 \\
\hline
\end{tabular}


The deconstruction of the forces in the $X$ direction for the calibration run is given by Fig. 8d. During the free vibration region the force due to vibration (dashed green line, given by Eq. 16) closely follows the transient force fluctuations measured using the balance ( $F_{x}$, solid blue line). The virtual and self mass terms attributable to the prescribed carriage motion can be calculated from Eq. (18) using the measure of instantaneous carriage acceleration $a_{\mathrm{car}}$. Subtraction of the vibration, virtual mass and self mass components from $F_{x}$ leaves the remainder force contribution (solid black line). There is only minor variation in the remainder force component after the velocity impulse. As we expect inertial force contributions to be dominant during free vibration, this indicates that the decomposition technique was successful. While some low pass filtering was still required to remove high-frequency vibration and electrical noise, it was possible to increase the cut-off frequency to approximately five times the first mode resonant frequency. In the following section, the velocity profile from the gust rig is characterised.

\section{Gust rig velocity profile}

PIV measurements of the flow exiting the outlet were taken to quantify the velocity profile and steadiness of the gust rig system.

\subsection{Steady state gust flow}

A time average of the flow field, taken over a $10 \mathrm{~s}$ period is shown in Fig. 9. On average, the velocity profile matches the ideal top-hat velocity profile well, however, the edges of the velocity profile spread due to the growth of the shear layer with increasing distance from the gust outlet $(y / c) .{ }^{1}$ Diffusion of vorticity in the shear layers either side of the gust is visible in Fig. 9a. The streaks of vorticity and corresponding fluctuations in the mean velocity profile inside the gust (from $0<x / c<2$ ) are due to the wakes of the turning vanes upstream of the gust rig outlet (see Fig. 3). This suggests that the flow through some of the diffusing turning vanes may have separated, or it may be attributable to thickening of the boundary layers as a consequence of the adverse pressure gradient. This will be the subject of a future investigation.

\subsection{Flow unsteadiness}

The unsteadiness of the cross flow region is quantified by the parameters $I_{u}=\frac{\sqrt{\overline{\overline{\left.u^{\prime}\right)^{2}}}}}{\bar{V}}$ and $I_{v}=\frac{\sqrt{\overline{{\left(\bar{v}^{\prime}\right)^{2}}^{2}}}}{\bar{V}}$, which is the ratio

\footnotetext{
${ }^{1}$ For an analytical expression of the gust shear layer velocity profile refer to Pope (2000), the plane mixing layer in chapter 5.
}

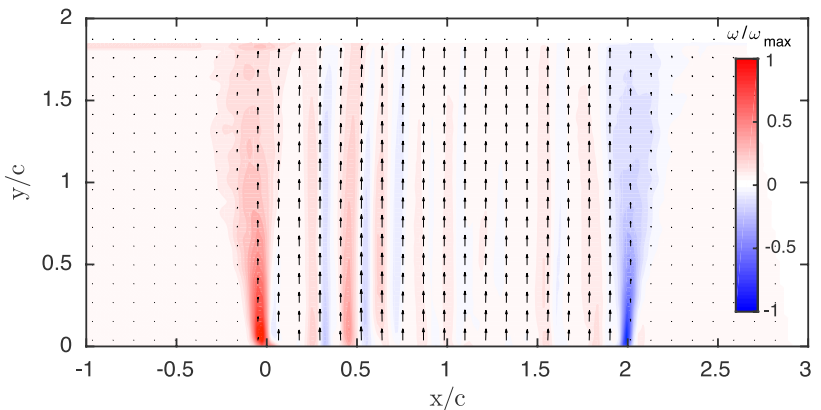

(a) Velocity quiver with contour of normalised vorticity

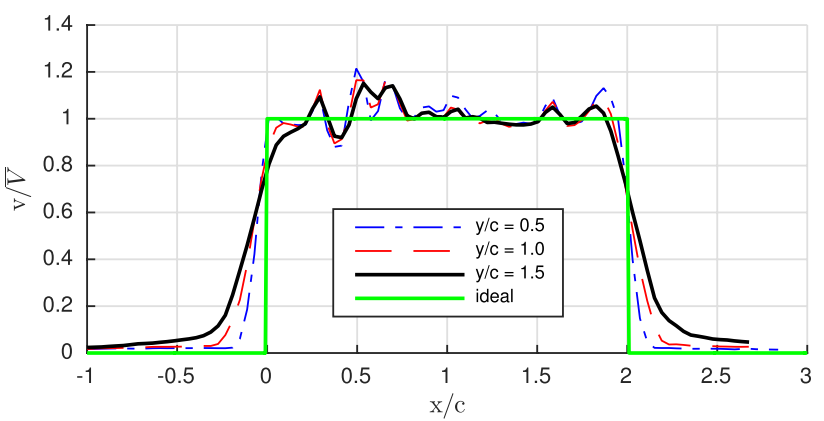

(b) Mean velocity profile

Fig. 9 Average flow velocity and normalised vorticity with position

of the average $\mathrm{u}$ and $\mathrm{v}$ component perturbation velocities $\left(u^{\prime}\right.$ and $v^{\prime}$, respectively) relative to the average gust velocity $(\bar{V})$. In Fig. 10, $I_{u}$ and $I_{v}$ are calculated from 1000 consecutive PIV frames (10 s) taken once the gust flow has reached steady state. This parameter is analogous to a turbulence intensity, however, unlike turbulence intensity the flow unsteadiness parameter represents a spatial average due to the size of each PIV interrogation window (Westerweel 1999). For the present setup, the side length of each processing interrogation window is $5.6 \mathrm{~mm}$ ( $4.6 \%$ chord), thus turbulent eddies with smaller length scales are not resolved.

Perturbations in the $u$ and $v$ velocities have a standard deviation of approximately $15-20 \%$ of the mean gust velocity within the gust shear layers. The unsteadiness peaks reduce in magnitude and width closer toward the gust outlet. At the centreline of the gust the perturbations are typically between 2 and 3\%, which is similar to the 'undisturbed' regions outside the gust.

\subsection{Theoretical difference in wing response between the real and ideal velocity profiles}

With the current configuration the gust rig generated a velocity profile that deviates from the ideal square wave profile, particularly at the shear layers. It is thus necessary to quantify the effect of these imperfections on the response of the wing. The Küssner function was used to calculate the response for each 


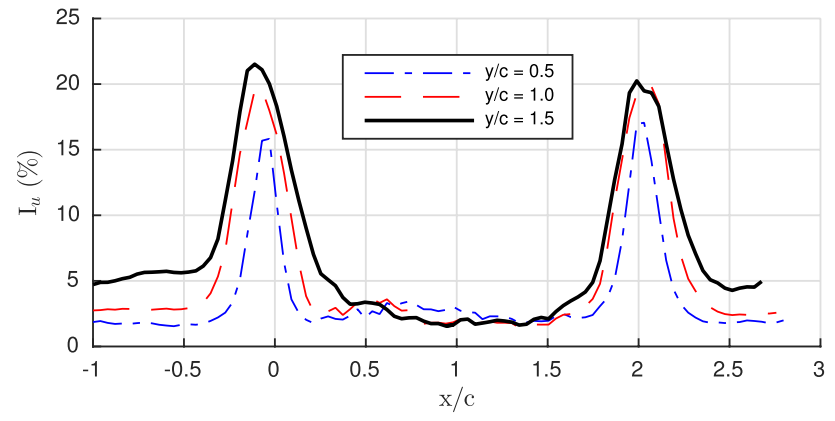

(a) u component

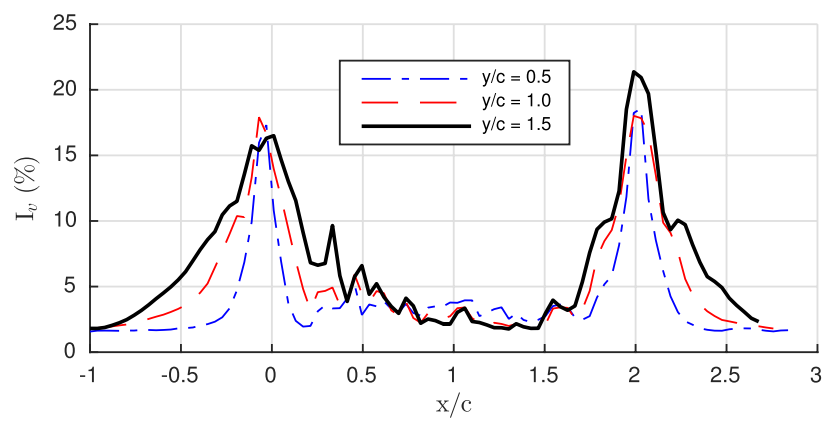

(b) $\mathrm{v}$ component

Fig. 10 Flow unsteadiness as a percentage of the mean gust velocity

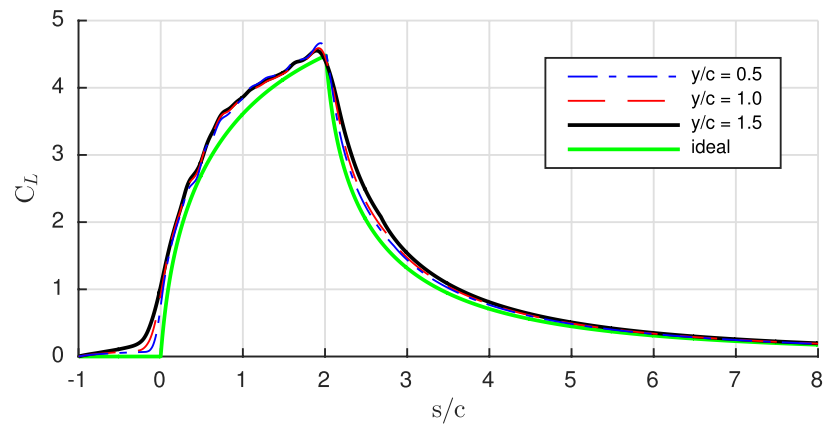

Fig. 11 Küssner response for each velocity profile given in Fig. 9b

profile shown prior in Fig. 9b. The change in lift coefficient is shown in Fig. 11 for $V / U=1$. The calculated responses thus follow the assumptions of Küssner's model highlighted in Sect. 1.1, which are shown later to only partly hold. Regardless, we see that the deviations from the ideal velocity profile have only a minor effect on the lift coefficient and the agreement is satisfactory at all $y / c$ locations.

\section{Wing-gust measurements}

Flow visualisation, PIV and force measurements were taken with the wing entering the cross flow at an offset of 1.5 chord lengths from the gust generator outlet plane. An extreme gust ratio of $V / U=1$ was chosen to identify dominant differences in flow topology between conditions experienced by UAVs in flight and those assumed with the Küssner model. While it was shown that reducing the distance between the gust outlet and wing increases both flow steadiness and the 'sharpness' of the shear layer, for these measurements the 1.5 chord length offset distance is used to reduce potential blockage effects due to the proximity of the ducting outlet to the wing. The Reynolds number for flow visualisation was 5000, while this was increased to 20,000 for PIV and 40,000 for force measurements. It has been shown by $\mathrm{Ol}$ and Babinsky (2016) that variations in Reynolds number within this range have little effect on the flow physics due to the clearly defined separation points at the leading and trailing edges of the flat plate. However, the relatively low Reynolds number used in the flow visualisation will increase the wavelength and thus the visual prominence of each roller of the Kelvin-Helmholtz and von Karman instabilities. Reynolds number dependence will be discussed further in Sect. 5.1 where the flow topology is considered.

\subsection{Flow topology}

Figure 12 shows flow features visualised by injecting a soy milk and methylated spirit dye mixture into the flow at both edges of the flat plate wing and in the shear layers either side of the gust outlet. This dye mixture was selected as its density can be varied to match the water in the tank by adjusting the milk/methylated spirit ratio. The dye was illuminated using the PIV laser which was defocussed to a nominal width of $40 \mathrm{~mm}$ to allow for illumination of dye that may have drifted in the span-wise direction. PIV measurements showing gradual entry of the wing into the gust are shown in Fig. 13.

From Figs. 12 and 13 the following can be observed:

- Prior to the wing entering the gust, the wing wake is visible as a von Karman vortex street. The wing sheds positive vorticity from the upper boundary layer and negative vorticity from the lower.

- As the wing enters through the gust shear layer the von Karman vortex street is replaced with a Kelvin-Helmholtz type instability. The flow visualisation shows that all wake vortices rotate in the clockwise direction, which is confirmed in the PIV measurements.

- After entering the gust, a leading edge vortex of anticlockwise sign has formed above the surfaced of the 


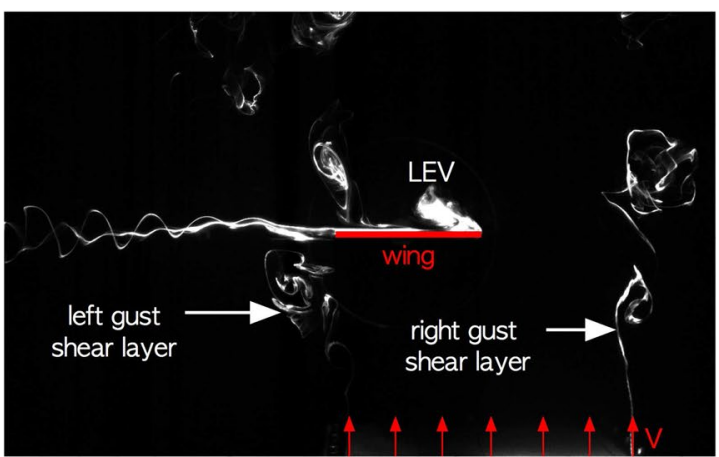

(a) flow visualisation at $\mathrm{s} / \mathrm{c}=1$

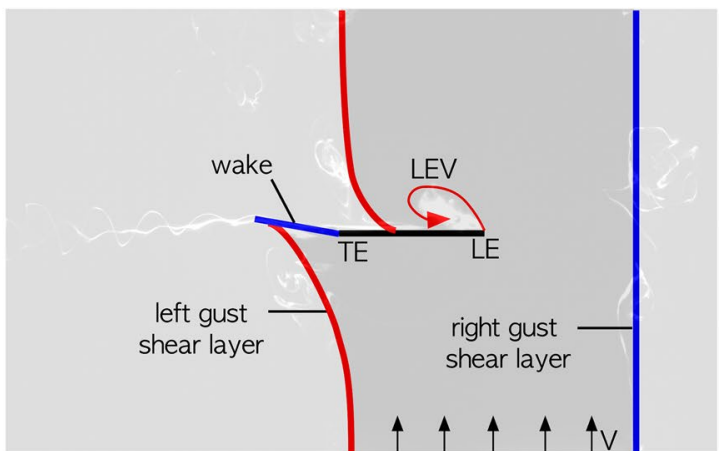

(b) flow schematic at $\mathrm{s} / \mathrm{c}=1$

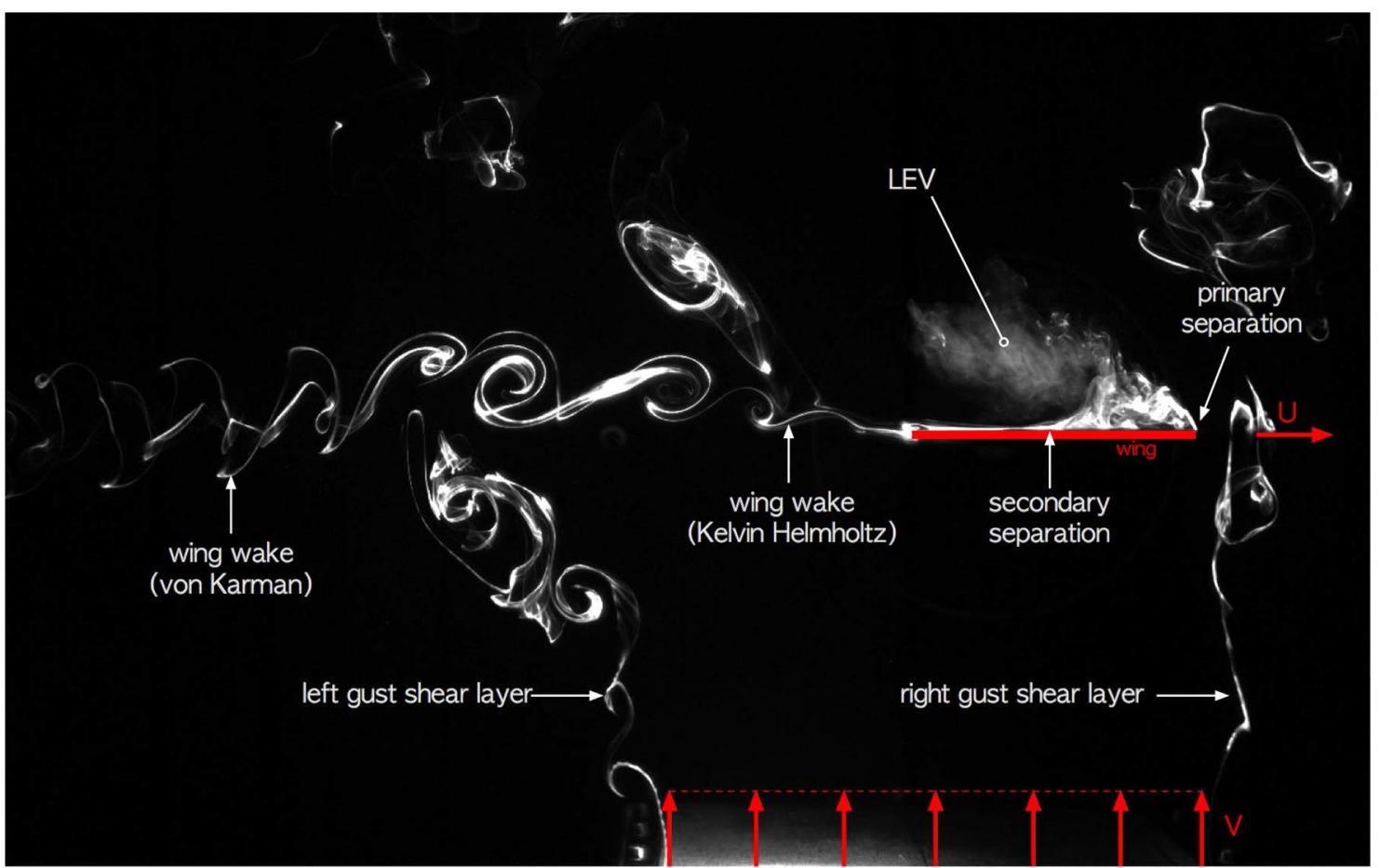

(c) flow visualisation at $\mathrm{s} / \mathrm{c}=2$

Fig. 12 Dye flow visualisation. Dye injection points are located at the leading and trailing wing edges and gust shear layers. $R e=5000$

wing. This is fed primarily by flow separating at the sharp leading edge.

- The vortex appears to induce flow along the upper surface of the wing, in a direction tangential to the wing velocity. At $s / c=1.5$ and onward, secondary separation on the upper surface of the wing is visible. The secondary flow appears to be entrained into the leading edge vortex.

- As the wing enters the gust, the left gust shear layer below the wing is diverted toward the wing's trailing edge. The left gust shear layer located above the wing is shifted in the direction tangential to the wing path.

It was assumed in the Küssner model that the wing has bound circulation, the wake is planar and the position of the gust shear layers is 'rigidly' held in space. For a gust ratio of 1 we see that the flow field is dominated by the leading edge vortex, which indicates that the bound circulation is comparatively reduced. Vorticity shed into the wake at the trailing edge is reasonably planar, however, the entry gust shear layer is notably deflected.

Each of these observations represents a deviation from the flow physics represented in the Küssner model, which suggests that there will be inaccuracies between the measured and modelled forces. In particular the presence of a leading edge vortex is ominous as the process whereby vorticity shed at the trailing edge regulates the growth of bound circulation is disrupted. With vorticity being shed at both edges there need not be any net bound circulation to conserve circulation. Consider a gust of infinite length. In 


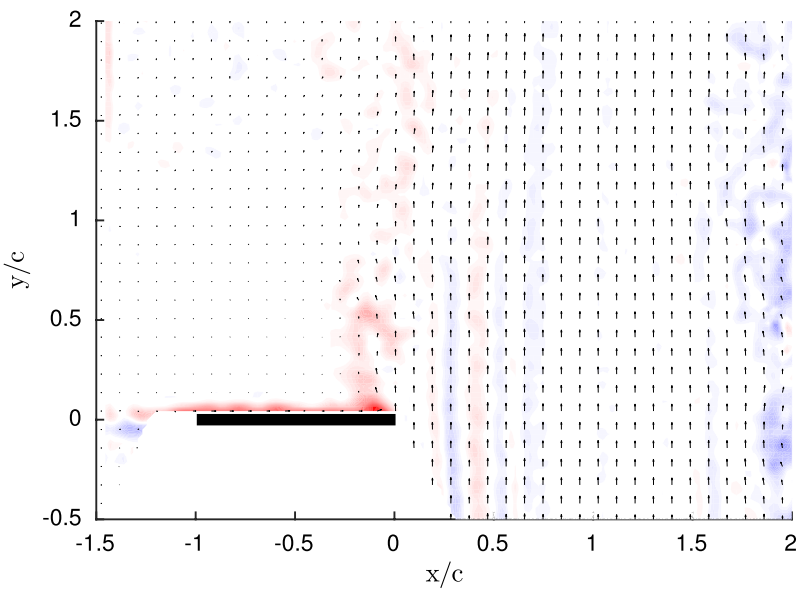

(a) $s / c=0$

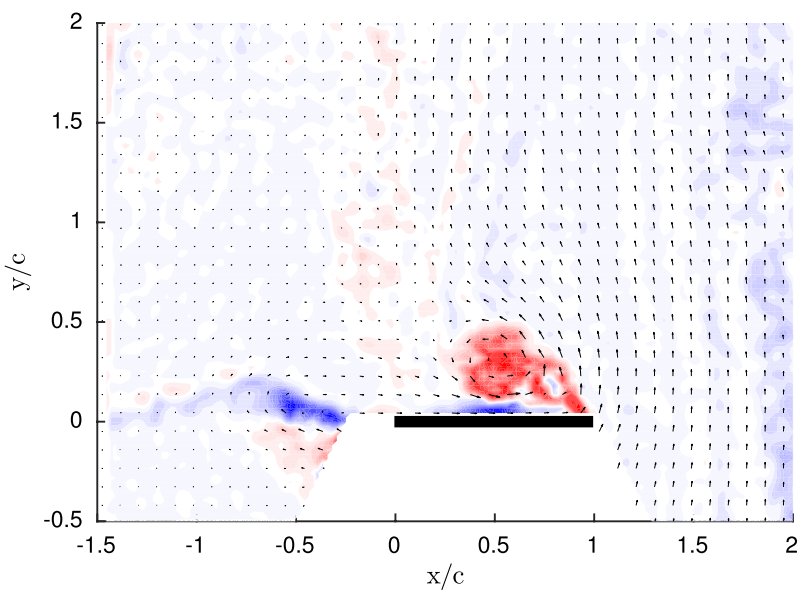

(c) $\mathrm{s} / \mathrm{c}=1$

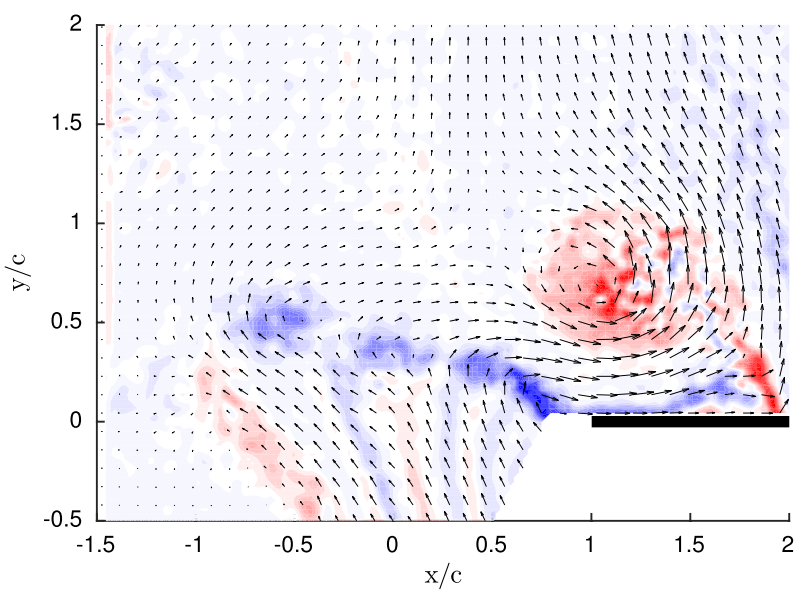

(e) $\mathrm{s} / \mathrm{c}=2$

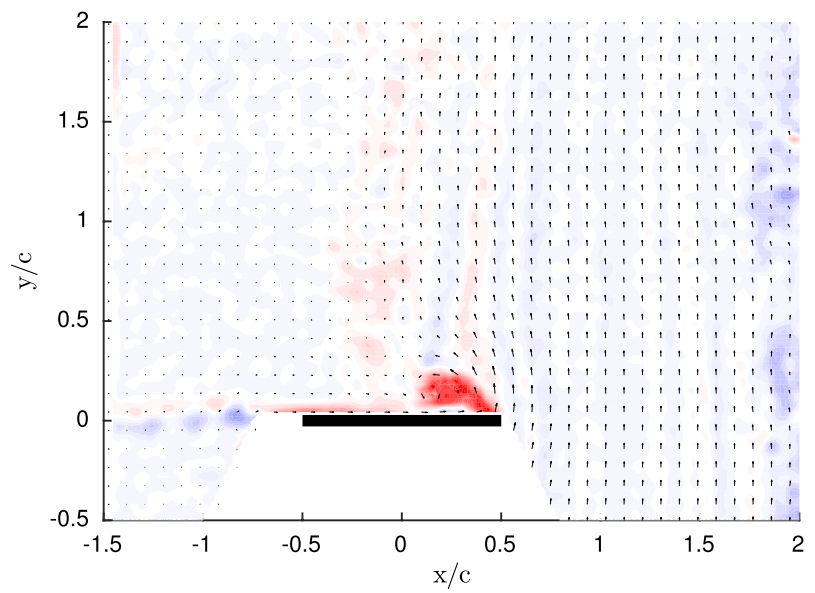

(b) $\mathrm{s} / \mathrm{c}=0.5$

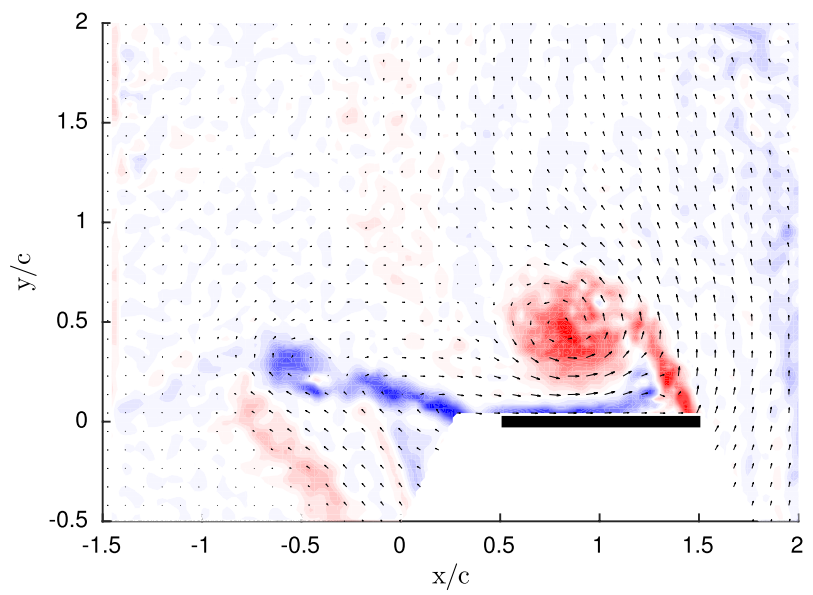

(d) $\mathrm{s} / \mathrm{c}=1.5$

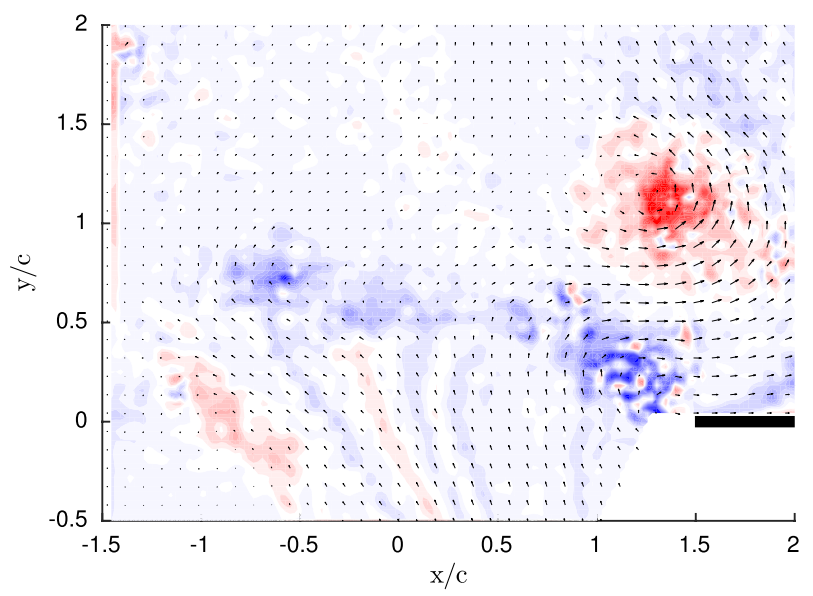

(f) $\mathrm{s} / \mathrm{c}=2.5$

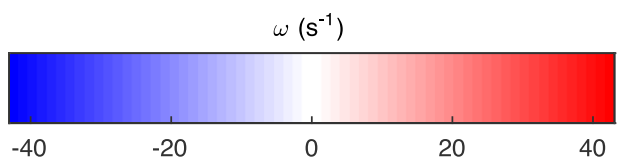

Fig. 13 PIV measurements of flow direction and vorticity during gust entry at $R e=20,000$. Every 4 th velocity vector is displayed 


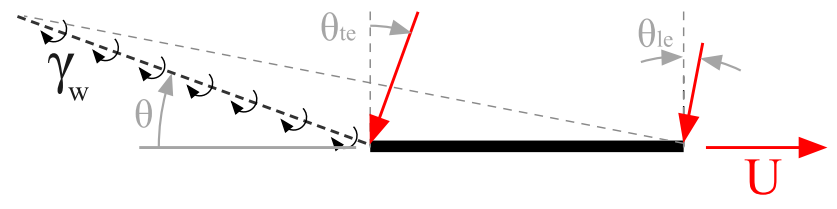

Fig. 14 Flow induced by the deflected wake at the leading and trailing edges

the Küssner model the mechanism in which lift is produced when at steady state is attributed to the relative advection of bound vorticity from a starting vortex at the free stream velocity. This gives constant lift, but no change in bound or shed circulation. For the high amplitude gust measured here there would be continual shedding of vorticity at both the leading and trailing edges, much like that of a surging plate at $45^{\circ}$ incidence. As Pitt Ford and Babinsky (2013) showed for such case, bound circulation is negligibly small.

The deflection of the wake will likely have a lower effect on the force response. Both the growth rate of bound circulation and the rate vorticity shed from each edge is dependent on the local flow velocity incident on the plate in the direction normal to the chord. With a wake deflection angle of approximately $\theta=20^{\circ}$ from the $x$ axis shown in Fig. 14 the downwash as illustrated would have a maximum deviation in the plate normal flow component of $\cos \left(20^{\circ}\right) \approx 0.94$ at the trailing edge, thus there is an approximately $6 \%$ difference due to the deflection angle. At the leading edge the angle is estimated to be less than $\theta_{\mathrm{le}}=10^{\circ}$, thus the flow normal velocity is reduced by less than approximately $1.5 \%$. This thus suggests that the effect of wake deflection on the force response is minor.

\subsubsection{Reynolds number effects}

It was argued at the beginning of Sect. 5 that the flow has little dependence on Reynolds numbers due to the clearly defined separation points at the leading and trailing edges. A comparison of flow topologies between Reynolds numbers of 20,000 and 40,000 is shown in Fig. 15. In both frames the leading edge vortex appears centred at an equivalent location above the wing and is of similar size. The vorticity shed at the trailing edge is additionally deflected at the approximately same angle. There is thus little qualitative difference in the flow topologies, thus suggesting that Reynolds number effects are small.

\subsection{Force measurements}

The prior analysis of flow topology suggests that the Küssner model will incorrectly predict the force response of the wing due to some significant differences in physics between the modelled and measured flows. Force measurements of the

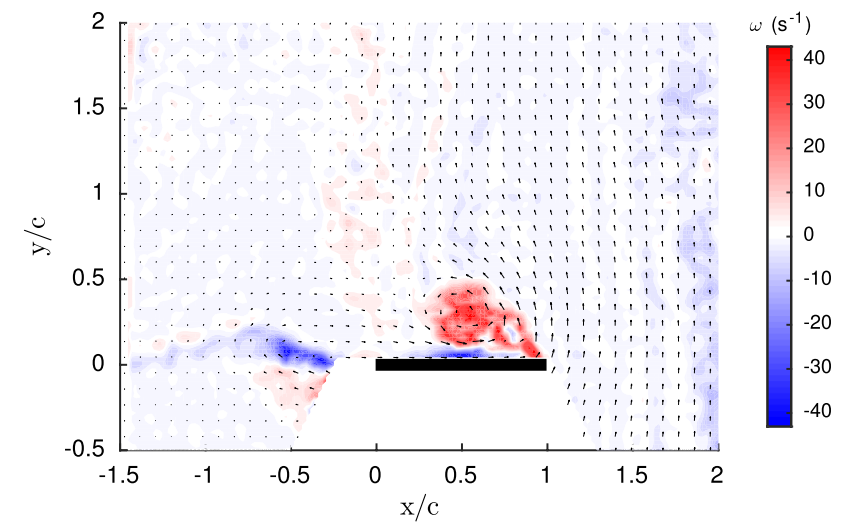

(a) $\operatorname{Re}=20,000$

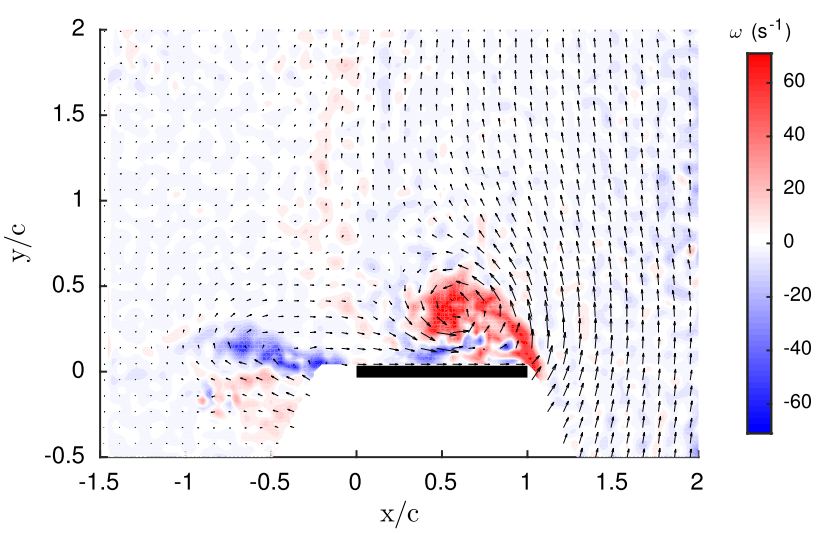

(b) $\operatorname{Re}=40,000$

Fig. 15 Comparison of flow topologies between Reynolds numbers of 20,000 and 40,000; gust ratio $1, s / c=1$. Each image is an average of 5 test runs

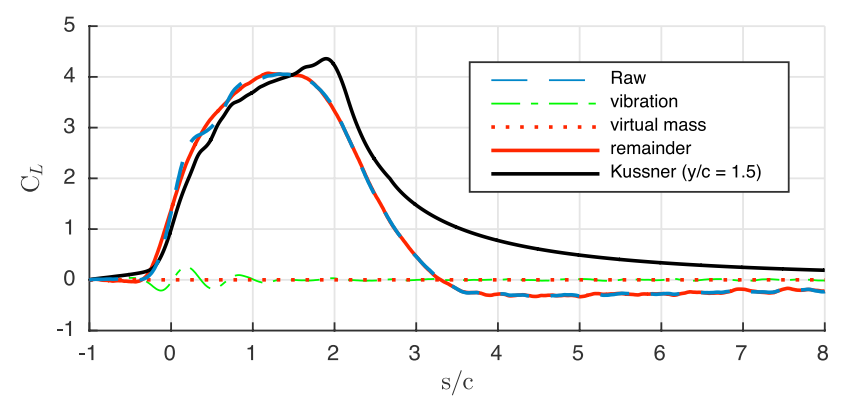

Fig. 16 Comparison between the experimentally measured lift with the Küssner response. $R e=40 \mathrm{k}$

gust encounter are shown in Fig. 16. It can be noted that the raw force measurement first rises prior to the nominal start of the gust $(s / c=0)$. This is a result of the thickened gust shear layer at the offset distance of 1.5 chord lengths (refer to Fig. 9). The raw force measurement has been deconstructed into vibration and virtual mass components as given in the methodology Sect. 3.3. As the wing is travelling at constant 
velocity through the gust, there is no virtual mass component that can be attributed explicitly by measurement of wing acceleration $\left(a_{\mathrm{car}}=0\right)$. There are minor oscillations in the raw force measurement between 0 and 1 chord length that can be attributed directly to wing vibration. In this instance, the vibration component is relatively minor compared to the full scale force, however, this is attributable to the favourable test conditions (high Reynolds number and gust ratio), but this will not generally be the case. Subtraction of the vibration and virtual mass force components gives the component of interest (remainder) for comparison with the Küssner response.

The measured lift coefficient and Küssner response match surprisingly well (within $\approx 10 \%$ ) during entry and up to $s / c=1.5$, after which discrepancies arise. The measured force overshoots $C_{\mathrm{L}}=0$ at $s / c=3.25$ and curiously generates negative lift for $s / c>3.25$. In contrast, the Küssner response indicates positive lift which asymptotes toward zero as $s / c \rightarrow \infty$. The initial close fit between the Küssner model and experiment is surprising given that the real flow field is dominated by the leading edge vortex, which is not accounted for in the Küssner model. If it is assumed that in the real flow field bound circulation is negligible, then the leading edge vortex must have equal and opposite circulation to the vorticity shed into the wake (assuming vorticity from the gust shear layer is not entrained into the LEV). From the flow visualisation the leading edge vortex appears to initially advect at a rate equal to the velocity of the wing (as a first order approximation). Thus in the Küssner model it may be possible to simply substitute the bound circulation with the leading edge vortex which may have similar dynamics. It is unknown why significant discrepancies between the theoretical and measured lift coefficients arise for $s / c>2$ as PIV measurements of the flow field have not been taken in this region. The analysis of flow topology in Sect. 5.1 showed that on entry of the plate in the gust severe distortion to the entrance shear layer occurs. It is thus likely that similar distortion occurs to the exit shear layer. This may provide an explanation as to why negative lift is measured on exit of the plate from the gust. When distorted the exit shear layer may roll up to form a coherent, clockwise rotating vortex. This vortex, along with the negative vorticity shed at the trailing edge of the wing (visible in Fig. 13f) would induce downwash onto the plate thus giving negative lift. For longtime periods the influence of these vortices would asymptote toward zero, thus a steady state lift coefficient of zero should eventually be reached.

\section{Conclusions}

This paper presents a unique cross flow test rig that was developed specifically to measure the response of aerofoils to large amplitude transverse gusts. An inertial system was additionally developed to reduce the effect of vibration contamination from force measurements. A single preliminary wing-gust encounter experiment with gust ratio of 1 was additionally conducted. PIV, flow visualisation, force and acceleration measurements indicate:

- The cross flow system can generate a sharp-edged jet into the towing tank with only minor disturbance to the surrounding fluid.

- Measurements of the velocity profile show reasonable uniformity across the gust width. Minor variations in the cross flow velocity profile are present due to possible separation on some vanes. Vane separation, along with an investigation into the span-wise uniformity, is to be investigated in a later study.

- The inertial force decomposition technique has been shown to allow the unsteady response of a model to be measured and compensated for, for frequencies up to that of the model's second structural mode of vibration.

- Entry of the wing into the gust region results in the formation of a large leading edge vortex and vorticity of the opposite sense is shed at the trailing edge.

- The trailing edge vorticity remains relatively planar, which fits the assumption of a planar wake in the Küssner theory.

- The entry gust shear layer is deflected upon wing entry.

- Despite differences between the physical and modelled flow fields, the lift coefficient predicted using the Küssner model fits measurements surprisingly well during entry into the gust. Significant discrepancies arise after $s / c=1.5$.

To conclude, the measurements for a flat plate wing encountering a transverse gust are certainly surprising, however, given only a single test case has been conducted it is unknown whether the trends can be applied generally or are isolated to this particular experiment. A full parametric study is outside of the scope of this work but is planned for the future.

Acknowledgements We would like to acknowledge the Cambridge Commonwealth European and International Trust, Churchill College and Schlumberger Limited for providing financial support.

Open Access This article is distributed under the terms of the Creative Commons Attribution 4.0 International License (http://creativeco mmons.org/licenses/by/4.0/), which permits unrestricted use, distribution, and reproduction in any medium, provided you give appropriate 
credit to the original author(s) and the source, provide a link to the Creative Commons license, and indicate if changes were made.

\section{References}

Adrian RJ, Westerweel J (2011) Particle image velocimetry. Cambridge University Press, Cambridge

Bisplinghoff RL, Ashley H, Halfman RL (1955) Aeroelasticity. Addison-Wesley series in mechanics. Addison-Wesley, Cambridge

Brion V, Lepage A, Amosse Y, Soulevant D, Senecat P, Abart JC, Paillart P (2015) Generation of vertical gusts in a transonic wind tunnel. Exp Fluids 56(145):1-16

Eldredge JD (2010) A reconciliation of viscous and inviscid approaches to computing locomotion of deforming bodies. Exp Mech 50(9):1349-1353

Friedman D, Westphal WR (1952) Experimental Investigation of a 90 degree Cascade Diffusing Bend with an Area Ratio of 1.45:1 and with Several Inlet Boundary Layers. TN 2668. Tech. rep., NACA. arXiv: $1011.1669 \mathrm{v} 3$

Graham WR, Pitt Ford CW, Babinsky H (2017) An impulse-based approach to estimating forces in unsteady flow. J Fluid Mech 815:60-76

Granlund K, Ol M, Bernal L (2011) Experiments on pitching plates: force and flowfield measurements at low Reynolds Numbers. In: 49th AIAA Aerospace Sciences Meeting

Ham ND, Bauer PH, Lawrence TL (1974) Wind tunnel generation of sinusoidal lateral and longitudinal gusts by circulation of twin parallel airfoils. Report CR-137547. Tech. rep., NASA

Homer MB, Saliveros E, Kokkalis A, Galbraith RAM (1993) Results from a set of low speed blade-vortex interaction experiments. Exp Fluids 14(5):341-352

Humphreys ND (1995) High Cross Wind Gust Loads on Ground from Moving Model Experiments. PhD thesis, University of Nottingham

Jones AR, Babinsky H (2010) Unsteady lift generation on rotating wings at low Reynolds numbers. J Aircr 47(3):1013-1021

Jones AR, Babinsky H (2011) Reynolds number effects on leading edge vortex development on a waving wing. Exp Fluids 51(1):197-210

Leishman JG (1996) Subsonic unsteady aerodynamics caused by gusts using the indicial method. J Aircr 33(5):869-879

Leishman JG (1997) Unsteady aerodynamics of airfoils encountering traveling gusts and vortices. J Aircr 34(6):719-729

Leishman JG (2000) Principles of helicopter aerodynamics, Cambridge aerospace series, vol 18, 2nd edn. Cambridge University Press, Cambridge

Mai H, Neumann J, Hennings H (2011) Gust response: a validation experiment and preliminary numerical simulations. In: 15th International Forum on Aeroelasticity and Structural Dynamics, pp 1-20

Mehta RD, Bradshaw P (1979) Design rules for small low speed wind tunnels. Aeronaut J R Aeronaut Soc 83:443-449

Mohamed A, Abdulrahim M, Watkins S, Clothier R (2015) Development and flight testing of a turbulence mitigation system for micro air vehicles. J Field Robot 33:639-660

Nobach H, Bodenschatz E (2009) Limitations of accuracy in PIV due to individual variations of particle image intensities. Exp Fluids 47(1):27-38

Ol M, Babinsky H (2016) Extensions of Fundamental Flow Physics to Practical MAV Aerodynamics: TR-AVT-202. In: Tech. rep, North Atlantic Treaty Organization

Patel MH (1982) Aerodynamic forces on finite wings in oblique gusts. J Aircr 19(1):71-75

Patel MH, Hancock GJ (1977) A gust tunnel facility. In: Tech. Rep. 3802, Aeronautical Research Council
Patel MH (1978) Aerodynamic forces on finite wings in oscillatory flow: an experimental study. AIAA J 16(11):1175-1180

Peng D, Gregory JW (2015) Vortex dynamics during blade-vortex interactions. Phys Fluids 27(5):1-23

Perrotta G, Jones AR (2017) Unsteady forcing on a flat-plate wing in large transverse gusts. Exp Fluids 58(8):1-11. https://doi. org/10.1007/s00348-017-2385-z

Pitt Ford CW (2013) Unsteady aerodynamic forces on accelerating wings at low Reynolds numbers. PhD thesis, The University of Cambridge

Pitt Ford CW, Babinsky H (2013) Lift and the leading-edge vortex. J Fluid Mech 720:280-313

Pope SB (2000) Turbulent flows. Cambridge University Press, Cambridge

Raffel M, Willert CE, Wereley ST, Kompenhans J (2007) Particle image velocimetry: a practical guide, 2 nd edn. Springer, Berlin

Rival D, Manejev R, Tropea C (2010) Measurement of parallel blade-vortex interaction at low Reynolds numbers. Exp Fluids 49(1):89-99

Ryan A, Dominy RG (2000) Wake surveys behind a passenger car subjected to a transient cross-wind gust. In: SAE World Congress, p 724. https://doi.org/10.4271/2000-01-0874

Saddington A, Finnis M, Knowles K (2014) The characterisation of a gust generator for aerodynamic testing. Proc Inst Mech Eng Part G J Aerosp Eng 229(7):1214-1225

Son O, Cetiner O, Stevens PRRJ, Babinsky H, Manar F, Mancini P, Jones AR, Ol MV, Gozukara AC (2016) Parametric variations in aspect ratio, leading edge and planform shapes for the rectilinear pitch cases of AVT-202. In: 54th AIAA Aerospace Sciences Meeting, pp 1-17

Spedding GR, Lissaman PBS (1998) Technical aspects of microscale flight systems. J Avian Biol 29(4):458-468

Stevens PRRJ (2013) Unsteady low Reynolds number aerodynamic forces. $\mathrm{PhD}$ thesis. The University of Cambridge

Stevens PRRJ, Babinsky H (2017) Experiments to investigate lift production mechanisms on pitching flat plates. Exp Fluids 58(1):7

Tang D, Dowell EH (2010) Aeroelastic airfoil with free play at angle of attack with gust excitation. AIAA J 48(2):427-442

Tang DM, Cizmas PGA, Dowell EH (1996) Experiments and analysis for a gust generator in a wind tunnel. J Aircr 33(1):139-148

Volpe R, Da Silva A, Ferrand V, Le Moyne L (2013) Experimental and numerical validation of a wind gust facility. J Fluids Eng 135(1):11106

Von Karman T, Sears WR (1938) Airfoil theory for non-uniform motion. J Aeronaut Sci 5(10):379-390

Walshe DEJ (1972) Wind-excited oscillation of structures. H.M, Stationery Office

Watkins S, Milbank J, Loxton BJ, Melbourne WH (2006) Atmospheric winds and their implications for microair vehicles. AIAA J 44(11):2591-2600

Watkins S, Thompson M, Loxton B, Abdulrahim M (2010) On low altitude flight through the atmospheric boundary layer. Int J Micro Air Veh 2(2):55-68

Westerweel J (1999) Fundamentals of digital particle image velocimetry. Meas Sci Technol 8(12):1379-1392

White C, Lim E, Watkins S, Mohamed A, Thompson M (2012) A feasibility study of micro air vehicles soaring tall buildings. J Wind Eng Ind Aerodyn 103:41-49

Zaide A, Raveh DE (2006) Numerical simulation and reduced-order modeling of airfoil gust response. AIAA J 44(8):1826-1834

Zanotti A, Ermacora M, Campanardi G, Gibertini G (2014) Stereo particle image velocimetry measurements of perpendicular bladevortex interaction over an oscillating airfoil. Exp Fluids 55(9):1-13

Publisher's Note Springer Nature remains neutral with regard to jurisdictional claims in published maps and institutional affiliations. 


\section{Affiliations}

\section{S. J. Corkery ${ }^{1}$ (I) H. Babinsky ${ }^{1}$. J. K. Harvey ${ }^{1}$}

$\triangle$ S. J. Corkery sjc276@cam.ac.uk
1 Department of Engineering, University of Cambridge, Trumpington Street, Cambridge CB2 1PZ, UK 